\title{
Multilevel Segmentation of Fundus Images using Dragonfly Optimization
}

\author{
S. Rakoth Kandan \\ Research Scholar \\ Computer Science and Engineering, \\ Annamalai University \\ INDIA
}

\author{
J. Sasikala \\ Assistant Professor \\ Computer Science and Engineering, \\ Annamalai University \\ India
}

\begin{abstract}
This paper presents a self adaptive dragonfly optimization (DFO) based methodology for performing multilevel segmentation of colour fundus images. The multilevel segmentation problem is formulated as an optimization problem and solved using the DFO. The method optimizes the threshold values for each of the three chromatic channels of colour fundus images through effectively exploring the solution space in obtaining the global best solution. The results of two fundus images illustrate the performance of the developed method.
\end{abstract}

\section{General Terms}

image processing, segmentation

\section{Keywords}

fundus images, multilevel segmentation.

\section{INTRODUCTION}

Retina is the inner part of human eye that senses the outside illumination. Light falls on retina and millions of optosensitive tissues convert these signals into electric signals and relay these signals to the brain for interpretation. Optical disc appears as an elliptical bright region on retinal fundus image, where all retinal blood vessels enter the retina. The pattern of blood vessels in retina are unique to a specific person which is important from the perspective of biometric analysis [1].

Diabetic retinopathy is the one among other main reasons of blindness in the adult population. Early discovery of diabetic retinopathy through screening programs and successive treatment is critical in order to avoid visual blindness. The early signs of diabetic retinopathy as manifested in retinal images include micro-aneurysms, hemorrhages and exudates. Clinicians commonly use retinal images for the screening differential diagnosis of retinal diseases such as retinal edema, diabetic retinopathy, age-related macular degeneration, malarial retinopathy, glaucoma, cataracts, exudates, lesions, prediction of strokes in hypertension patients and so on [2-4].

The increasing pervasiveness of diabetes and low number of clinical specialists, increase the need for automatic methods to reduce the workload on physicians [5] besides making the diagnosis robust and consistent. Color fundus imaging has emerged as the preferred procedure by the medical community for comprehensive large-scale retinal disease screening due to their ease of acquisition and good visibility of retinal structures [6].

In the literature, a number of methods for segmenting the optical disk, blood vessels, exudates, cataracts, etc have been suggested [7-12]. Most of these methods consider the gray level representation or a single color plane in segmenting the fundus images, rather than taking into account the multiple color channels of fundus images. For instance, the contrast enhanced $\mathrm{L}$ channel of $\mathrm{L} * \mathrm{U}^{*} \mathrm{~V} *$ color spaces has been used to apply morphological operations as well as H-maxima transform for detecting exudates in [7]. The gray level image has been thresholded to segment optic disc and exudates in retinal images in [10]. A geometrical model of vessel structure involving gray scale image has been suggested for detection of optic disc in retinal images in [11]. In [12], the grey level version of the color original image has been used to segment the optical disk on two of the three different approaches presented: multi-thresholding and active contour without edges.

It is well known fact that the segmentation of color image demonstrates to be more useful than the segmentation of gray scale image, because color image expresses much more image features than gray scale image. In fact, each pixel is characterized by a great number of combination of R, G, B chromatic components. The segmentation of fundus images would have been more effective, if segmentation is performed considering the rich chromatic information in all the three color channels of fundus images. However, it requires the computational cost considerably higher than that needed for gray scale image, but it is no longer a major problem with the increasing speed of computation. In fact, there has been a remarkable growth of techniques for the segmentation of color images in this decade $[13,14]$.

Recently, a dragonfly optimization (DFO), a population based meta-heuristic optimization algorithm that simulates the static and dynamic swarming behaviors of dragonflies [15], has been applied for performing multilevel segmentation of gray scale images [16]. The multilevel segmentation problem has formulated as an optimization problem and solved using the DFO. The method optimizes the threshold values through effectively exploring the solution space in obtaining the global best solution.

The focus of this paper is to develop a multilevel thresholding based method for segmenting the fundus images in RGB colour space by modifying the DFO based gray scaled segmentation method. The paper is organized with four sections containing introduction, proposed method (PM), results and discussions, and conclusion.

\section{PROPOSED METHOD}

The threshold-based methods assume that an image is composed of regions with different intensity ranges. The optimum threshold values are determined by the histogram of the image which has the valleys between two adjacent peaks [16]. Owing to its simplicity, histogram thresholding is a widely used technique for gray scale image segmentation. But it is not a trivial job for color image because of its multidimensional structure $[13,14]$. The PM is an extension of the 
segmentation method suggested by the authors in [16] with a view of processing the $\mathrm{R}, \mathrm{G}, \mathrm{B}$ chromatic components of fundus images. The median filter is applied for noise removal.
The proposed method involves representation of decision variables and formation of a fitness function.

Table 1 Results for Fundus Image-1

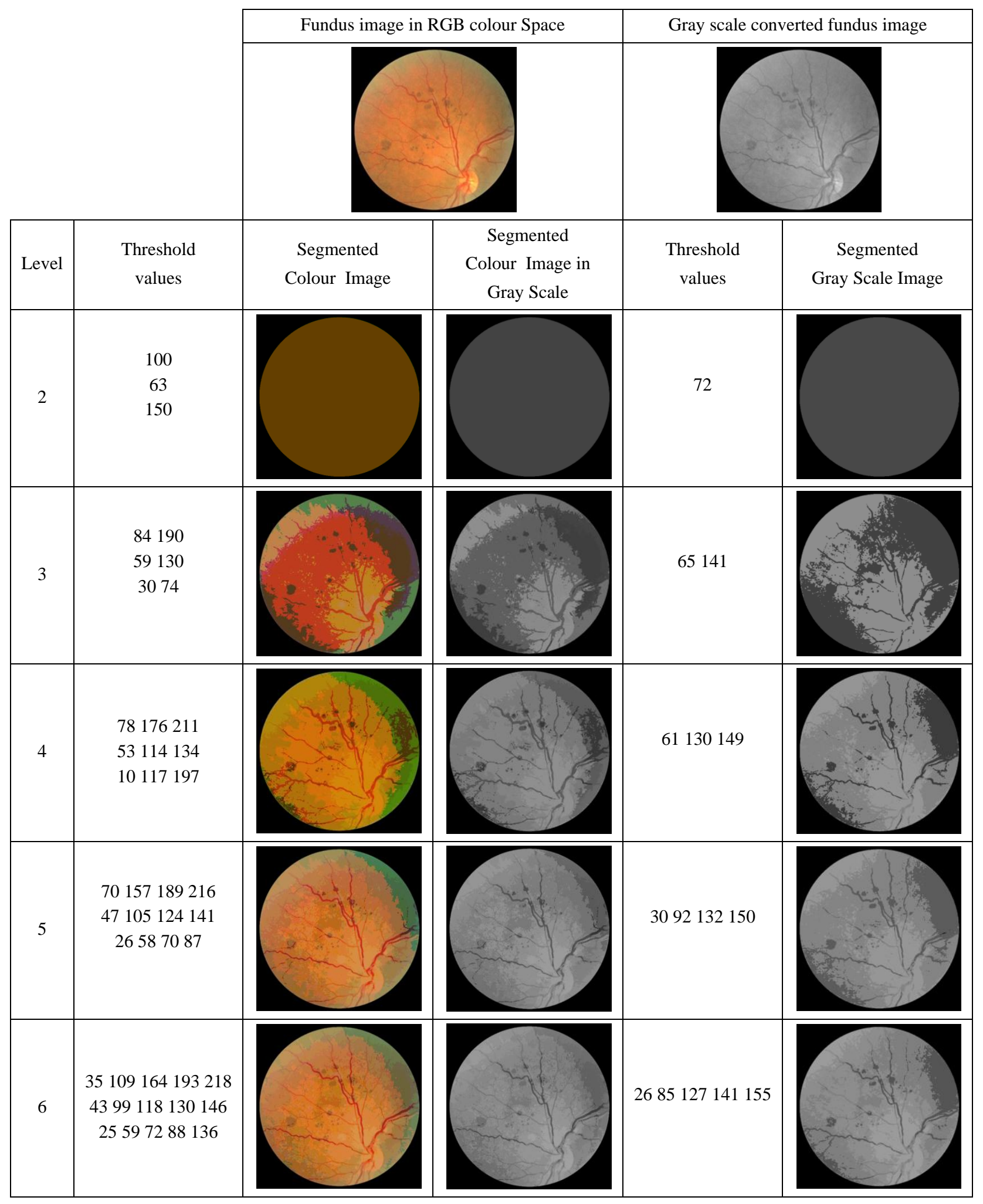


Table 2 Results for Fundus Image-2

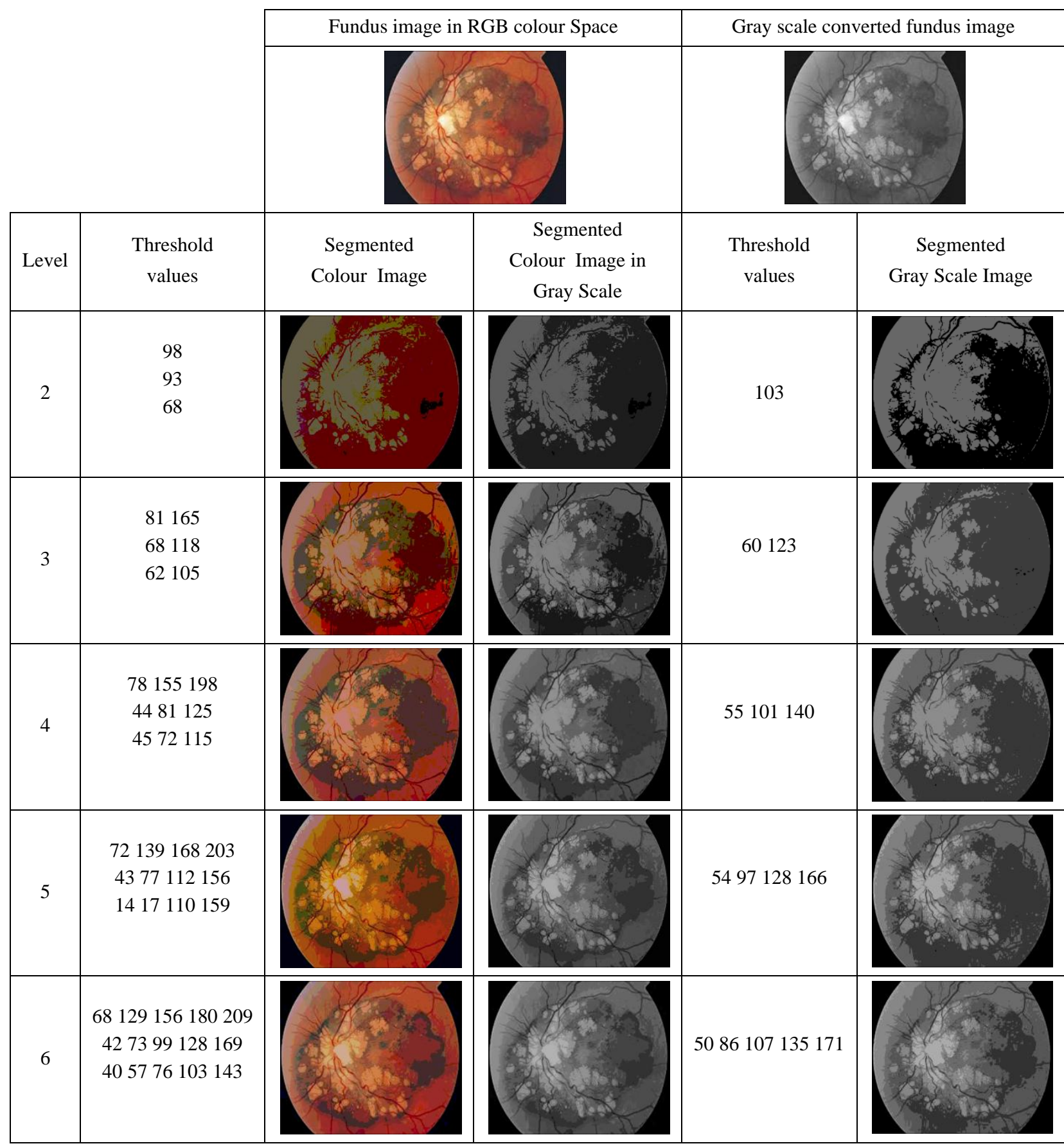

Each chromatic channel of RGB fundus image is divided into $n c$ number of classes by $n c-1$ number of thresholds of $\left\{T_{1}, T_{2}, \cdots, T_{n c-1}\right\}$. These thresholds act as separators between the consecutive classes of $\left\{C_{1}, C_{2}, \cdots, C_{n c}\right\}$ in the range of threshold values of $\left\{\left[0, \cdots, T_{1}\right],\left[T_{1}+1, \cdots, T_{2}\right], \cdots \cdots,\left[T_{n c-1}+1, \cdots, L\right]\right\} \quad$ for $\quad$ each chromatic channel, where $\mathrm{L}$ is the maximum pixel intensity value of the digital image. In the PM, each dragonfly $d f$ is defined to denote the threshold levels of all the three colour spaces and the self-adaptive parameters as decision variables as

$$
d f=\left\{\begin{array}{l}
T_{1}^{R}, T_{2}^{R}, \cdots, T_{n c-1}^{R}, \\
T_{1}^{G}, T_{2}^{G}, \cdots, T_{n c-1}^{G}, \\
T_{1}^{B}, T_{2}^{B}, \cdots, T_{n c-1}^{B}, \\
s_{i}, a_{i}, c_{i}, f_{i}, e_{i}, \gamma_{i}, \omega_{i}
\end{array}\right\}
$$

The SADFO searches for optimal threshold values by maximizing a fitness function $F$. based on Kapur's entropy.

$$
\text { Maximize } F=\sum_{\text {colou } r \in R, G, B}\left\{\sum_{k=1}^{n c} H_{k}^{\text {colour }}\right\}
$$

Where $H_{k}^{\text {colour }}$ represents $k$-th entropy of the selected colour channel of RGB image and is evaluated by 


$$
\begin{aligned}
& H_{1}^{\text {colour }}=\sum_{i=0}^{T_{1}^{\text {colour }}} \frac{p_{i}}{\chi_{1}} \ln \left(\frac{p_{i}}{\chi_{1}}\right) \quad ; \quad \chi_{1}=\sum_{i=0}^{T_{1}^{\text {colour }}} p_{i} \\
& H_{2}^{\text {colour }}=\sum_{i=1+T_{1}^{\text {colour }}}^{T_{2}^{\text {colour }}} \frac{p_{i}}{\chi_{2}} \ln \left(\frac{p_{i}}{\chi_{2}}\right) ; \quad \chi_{2}=\sum_{i=1+T_{1}^{\text {colour }}}^{T_{2}^{\text {colour }}} p_{i} \\
& H_{n c}^{\text {colour }}=\sum_{i=1+T_{n c-1}^{\text {colour }}}^{L} \frac{p_{i}}{\chi_{n c}} \ln \left(\frac{p_{i}}{\chi_{n c}}\right) ; \quad \chi_{n c}=\sum_{i=1+T_{n c-1}^{\text {colour }}}^{L} p_{i} \\
& \text { colour } \in\{R, G, B\}
\end{aligned}
$$

$p_{i}^{\text {colour }}$ represents probability distribution at $i-t h$ intensity level of the selected chromatic channel of RGB image and is calculated by

$p_{i}^{\text {colour }}=\frac{h_{i}^{\text {colour }}}{n p} ; \quad i \in\{0,1, \cdots, L\} ;$ colour $\in\{R, G, B\}$

$h_{i}^{\text {colour }} \quad$ indicates number of pixels that corresponds to $i-t h$ intensity level of the selected chromatic channel of R, G and B.

$n p \quad$ is the total number of pixels in the image.

$\chi_{i} \quad$ denotes the probability of set $C_{i}$

An initial swarm of dragonflies is obtained by generating random values within their respective limits. The fitness function $F$ is calculated by considering the threshold values of each dragonfly; and the exploration and exploitation phases, which represent social interaction of dragonflies in navigating and searching for foods and avoiding enemies, are performed for all the dragonflies in the swarm with a view of maximizing their finesses. The iterative process is continued till convergence [16].

\section{RESULTS AND DISCUSSIONS}

The PM has been tested on two fundus images. As the images are rectangular shaped with different sizes, the width of these images is adjusted to have 512 pixels and the height is proportionally altered with a view to have the true shape of the images. The software package is developed in Matlab platform and executed in a $2.67 \mathrm{GHz}$ Intel core-i5 personal computer. The results of the PM are compared with that of the gray scale based existing method (EM) suggested in [16] with a view of studying the performances.

The results for threshold levels of 1,2, 3, 4 and 5 are obtained and presented in Table 1 and 2. These table also include the original RGB and gray scale converted fundus images. The threshold values for the PM are given in second column of the tables, while for the EM, they are given in the fifth column of the tables. It can be observed that the segmented colour image, given in third column of the table, conveys more accurate information than that of the segmented gray image given in the last column of the table. The segmeted colour images are also converted into gray scale images and presented in fourth column of the tables. The visual comparison of gray scale images of the PM with those of the EM also ensures that the PM is able to make better segmentation. The visual analysis of these results clearly indicate that the segmented results are better with more number of threshold levels.

\section{CONCLUSIONS}

DFO is a population based optimization algorithm that simulates the static and dynamic swarming behaviors of dragonflies. A self adaptive DFO based methodology for performing multilevel segmentation of colour fundus images has been presented. The multilevel segmentation problem has been formulated as an optimization problem and solved using the self adaptive DFO. The method has been applied on two fundus images with a view of illustrating the performances. It has been found from the results that the PM effectively yields better segmented results than that of performing segmentation after converting the image into gray scale. The method can be modified to classify various retinal diseases such as retinal edema, diabetic retinopathy, age-related macular degeneration, malarial retinopathy, glaucoma and cataracts, exudates.

\section{ACKNOWLEDGMENTS}

The authors gratefully acknowledge the authorities of Annamalai University for the facilities offered to carry out this work.

\section{REFERENCES}

[1] Hill, Robert Buzz. (1999). "Retina Identification" from the Book: Biometrics: Personal Identification in Networked Society, 1st edn. Springer, Berlin.

[2] T. Chui, M. Dubow, A. Pinhas, N. Shah, A. Gan, R. Weitz, Y. Sulai, A. Dubra, R. Rosen. (2014). Comparison of adaptive optics scanning light ophthalmoscopic fluorescein angiography and offset pinhole imaging, Biomed. Opt. Express, 5(4): 11731189.

[3] N. Patton, T. Aslam, T. MacGillivray, A. Pattie, I. J. Deary, B. Dhillon. (2005). Retinal vascular image analysis as a potential screening tool for cerebrovascular disease: a rationale based on homology between cerebral and retinal microvasculatures, J Anat., 206: 319-348.

[4] M. Potchen, S. Kampondeni, K. Seydel, G. Birbeck, C. Hammond, W. Bradley. (2012). Acute brain mri findings in 120 malawian children with cerebral malaria: new insights into an ancient disease, Am. J Neuroradiol., 33: 1740-1746.

[5] S. Philip, AD. Fleming, KA. Goatman, S. Fonseca, P. Mcnamee, GS. Scotland, GJ. Prescott, PF. Sharp, J.A. Olson. (2007). The efficacy of automated disease/nodisease grading for diabetic retinopathy in a systematic screeningprogramme, Br. J. Ophthalmol., 91: $1512-1517$

[6] Daniel E. Singer, David M. Nathan, Howard A. Fogel, and Andrew P. Schachat. (1992). Screening for diabetic retinopathy, Annals of Internal Medicine, 116(8): 660 671.

[7] D. Welfer, J. Scharcanski, D.R. Marinho. (2010). A coarse-to-fine strategy forautomatically detecting exudates in color eye fundus images, Comput. Med.Imaging Graph, 34: 228-235.

[8] S. Ali, D. Sidibé, K.M. Adal, L. Giancardo, E. Chaum, T.P. Karnowski, F.Mériaudeau. (2013). Statistical atlas based exudate segmentation, Comput. Med.Imaging Graph, 37: 358-368.

[9] B. Harangi, A. Hajdu. (2014). Automatic exudate detection by fusing multiple active contours and 
regionwise classification, Comput. Biol. Med., 54: 156171.

[10] Kavitha, D., Shenbaga Devi, S. (2005). Automatic detection of optic disc and exudates in retinal images, in: Proceedings of 2005 International Conference on Intelligent Sensing and Information Processing, 501506.

[11] Foracchia, M., Grisan, E., Ruggeri, A. (2004). Detection of optic disc in retinal images by means of a geometrical model of vessel structure. IEEE Transactions on Medical Imaging, 23: 1189-1195.

[12] Madhusudhan M., Malay N., Nirmala S.R., Samerendra D. (2011) Image Processing Techniques for Glaucoma Detection. In: Abraham A., Mauri J.L., Buford J.F., Suzuki J., Thampi S.M. (eds) Advances in Computing and Communications. ACC 2011. Communications in Computer and Information Science, Springer, Berlin, Heidelberg, 192: 365-373.
[13] Guo Dong, and Ming Xie. (2005). Color Clustering and Learning for Image Segmentation Based on Neural Networks, IEEE Trans on Neural Networks, 16(4): 925935 .

[14] Tahir Sag and Mehmet Cunkas. (2015). Color image segmentation based on multiobjective artificial bee colony optimization, Applied Soft Computing, 34(C): 389-401.

[15] Seyedali Mirjalili. (2015). Dragonfly algorithm: A new meta-heuristic optimization technique for solving singleobjective, discrete and multi-objective problems, Neural Comput and Applic. DOI. 10.1007/s00521-015-1920-1.

[16] Rakoth Kandan Sambandam, Sasikala Jayaraman. (2016). Self-Adaptive Dragonfly Based Optimal Thresholding for Multilevel Segmentation of Digital Images, Journal of King Saud University - Computer and Information Sciences, http://dx.doi.org/10.1016/j.jksuci.2016.11.002. 